\title{
Part VIII: Summaries and Conclusions
}

L. SPITZER, JR., Princeton Observatory, Princeton, New Jersey: In this final session, of both the present Symposium and the series of three symposia, we attempt to ask: What do we really know about the dynamics of cosmic clouds? We have first a series of impressions and summarizing remarks, then a general discussion.

J. M. BURGERS, University of Maryland, College Park, Maryland. With the progress of our meetings, it has become more and more difficult to give a proper summary immediately at the end of the meeting itself, because the subject is being explored into deeper regions, more details have come up, and the number of questions is continually increasing. This is the reason why the word summary is possibly best replaced today by "impressions."

One impression which probably all of you will have had, is the remarkable continuity of these meetings, notwithstanding the intervals of four years between them. Apparently the subject as originally proposed was conceived so well that it continually evokes fresh interest. New details have been added and the picture in our minds has evolved, but we have not lost sight of our original problems and unfortunately we must say that the same problems are still before us.

If I look back over the previous Symposia, as a basis for comparison with the present one, I believe that in 1949 we were haunted by the problem of turbulence as possibly having some meaning for the motion of the interstellar gas. Much thought was given to the question whether the differential rotation of the galaxy could be the source of the turbulence, and whether turbulence would lead to a transfer of energy from large scale to smaller scale motion and finally to dissipation. Turbulence was then mainly considered from the point of view which had been developed in the theory of incompressible flow. An important item in that Symposium was von Karman's survey of the theory in which he mentioned Kolmogoroff's law relating the average difference of speeds at two points in a turbulent system, to the distance between these points. This relation became a regular feature in the analysis of astronomical data. A question which baffled us was whether the turbulence as it was conceived at that time could lead to the appearance of the large density variations, which are observed in the interstellar gas, in a ratio from 1 to 100 , and which play an important part in all astronomical considerations. The recognition of these large density variations brought us to a discussion of expansion waves and shock waves in the gas. At the same time the importance of magnetic forces was recognized, and Batchelor discussed the relations existing between magnetic fields and velocities in a turbulent medium of large electronic conductivity. The magnetic fields formed somewhat a sideline, although an important one.

In 1953, during the Second Symposium, new points of view came up. In the first place, the optical radiation from shock waves was extensively discussed by Kantrowitz from an empirical standpoint. Much experimental work had been done in laboratories on this radiation, and the possible relation of the phenomena observed in shock tubes to the bright rims often presented by interstellar gas clouds was brought to the foreground. In the second place, Oort spoke about a mechanism for the production of large density variations in the interstellar gas, due to the radiation from very hot stars, which brings enormous amounts of energy into the gas surrounding the star. Along with the production of energy, the possibilities for getting rid of it were discussed. Zanstra called attention to the existence of peculiar cooling mechanisms, depending on the presence of ions with particular energy levels. The data obtained from theoretical calculations by Spitzer and Savedoff were reviewed. This brought very clearly to our minds how closely astrophysical problems are related to the problems of atomic radiation theory. Although the details of the mechanisms involved were not settled, it became evident that the form of turbulence observed in the interstellar gas with its large density fluctuations owes its peculiarities to the circumstance that on the one hand there exist randomly distributed sources of energy to replenish the field of mechanical motions, and, on the other hand, there are mechanisms coupling the local dissipation of mechanical motions to radiation fields which disperse the dissipated energy from the immediate neighborhood.

Coming finally to the present Symposium, we must confess that most of the old problems are still before us. Compressible turbulence has not yet been developed as a theoretically well-understood subject. To the question whether the differential rotation of the galactic gas can be a source of turbulence, no further answer has been given than what was summed up by Batchelor in yesterday's session: the main criterion is whether the circulation around any circular orbit is increasing with the distance from the center or not. A theory concerning the stability or the instability of motion with differential rotation, taking into account some form of viscosity, and having a gravitational field to keep the gas together instead of confining walls, has not been developed. My point of view is that the differential rotation does not form a likely source of energy for the observed form of compressible turbulence; I would believe that the source of energy is to be found in the nuclear reactions in stars, and that the nature of the turbulence is due to randomness in the distribution of radiating stars in 
space and time, combined with the existence of effective cooling mechanisms, and the effects of compression and gravitation in producing new centers for nuclear reactions.

In the meantime, the observational data on turbulence in the interstellar gas and the data on the features presented by bright rims have been extended and brought to much greater precision. Quantitative data on the energy balance have been reviewed and refined. Seaton's account concerning cross sections for various reactions is of great importance in connection with the energy dissipation by inelastic excitation and radiative emission.

The picture of the over-all structure of the interstellar gas has been clarified. Formerly, we just spoke about interstellar clouds; today we have seen that these clouds must be located in the spiral arms of the galaxy. In view of the data obtained from observations on the $21-\mathrm{cm}$ radiation, we now must ask: is a spiral arm perhaps a single cloud, or is it still a collection of clouds? And if so, do clouds collide in the spiral arms? Can they get out of a spiral or is their motion confined in some way? In this picture, the magnetic field has obtained a much more fundamental place, for it seems that the pressure due to the magnetic force may confine the interstellar gas in the spiral arms.

It has been recognized that there is a somewhat spherical or ellipsoidal "halo" of gas around the galaxy. It has been noted that motions of the gas in the galaxy proper will provoke expansion waves or shock waves traveling through this halo. Hence, the energy balance of the interstellar gas has received a new term: there can be loss of energy to the halo; perhaps it may be lost from the gas in the halo by radiation. Also, in these problems, the magnetic field appears to play a part as it is involved in the equilibrium of the gas in the halo.

In this way, the Symposium has brought an extension of the picture which we could not foresee in the preceding symposia. Although in yesterday's session, it was decided that future symposia will give attention primarily to stellar atmospheres, I do not doubt that many of you will keep in mind the new and interesting picture of the galaxy with its halo, and you will have received a fresh inducement to think about magnetic fields and their influence on gas motion.

H. W. LIEPMaNN, California Institute of Technology, Pasadena, California: The previous conferences, in Paris and in Cambridge, England, differed somewhat from the present one. In both previous meetings, I felt that a specific gas-dynamical phenomenon was "discovered" and an attempt made to apply it to a host of different astrophysical situations. In Paris, this was turbulence, in Cambridge, shock waves. The present meeting looks to me much more balanced. Some diffusion of gas dynamics into astrophysics and of astrophysics into gas dynamics has evidently taken place, and it seemed to me that fewer rash statements and jumpy conclusions from both sides were made at this conference. Since this diffusion of interest and knowledge was the primary aim of the conferences, the string of meetings was evidently successful. As far as my own interest goes, I was struck by the increasing ability of defining gas-dynamical problems of astrophysical importance and of the possibility to make laboratory experiments on these problems.

In general, we deal with the dynamics of an ionized gas in the presence of radiation and of magnetic fields. Consequently, all possible interactions of the different fields, i.e., turbulence, the pressure field, and the electromagnetic fields are important. Thus, for example, the interaction between the first two are characteristic of turbulence in a compressible fluid. The interaction between vorticity and the electromagnetic field leads to the question of the spontaneous appearance of random magnetic fields. Finally, the interaction between pressure waves and electromagnetic fields leads to a much greater variety in possible shock solutions than in the usual gas dynamics.

All interactions with electromagnetic fields depend very much on the electrical conductivity of the medium. Laboratory experiments seemed nearly impossible at the time of the Paris meeting, since the techniques of obtaining very high temperatures and thus, large conductivities were not yet developed. At the Cambridge meeting, Kantrowitz presented his shock tube techniques of reaching strong shock waves and high temperatures. In Cambridge, a shock Mach number of the order of 10 and temperatures of $10^{4}$ degrees was considered high. In the present meeting, only four years later, work on shock waves with Mach numbers of about 200 and temperatures of the order of $10^{6}$ was reported. Evidently we have now at least the possibility of studying astrophysical gas dynamics on a laboratory scale. In this connection, I would recapitulate just a few problems raised and not settled at this conference which could eventually be studied.

In Petschek's contribution, the problem of the flow within a shock wave in a conducting fluid in the presence of strong magnetic fields was raised. The study of shock waves under such conditions has barely started, and the whole field of pressure waves in ionized media now appears to me a wide open field for laboratory experiments.

Wilson's paper on planetary nebulae suggests the study of expanding gas spheres into very low pressure regions, possibly in the presence of magnetic fields. Here, too, ingenious experiments on a laboratory scale do not seem completely out of reach.

Münch's and also Pickelner's studies of fluctuation spectra in nebulae have shown the - not unexpectedinadequacy of incompressible turbulence considerations for the motion in nebulae. At present, the subject of turbulence in compressible fluid flow has hardly been 
studied systematically. From laboratory experimentsand daily experiments in life - we know a little about the production of sound waves from turbulence, a subject which was discussed from the theoretical side by Lighthill in Cambridge. However, we have almost no laboratory experience with flows in which the energy content in the turbulence and in the shock wave field is comparable. To get into this range, one will have to reach turbulent fluctuation velocities of the order of the velocity of sound. Since turbulent velocity fluctuations have magnitudes of roughly $10 \%$ of the mean velocities, mean velocities of the order of 10 times the velocity of sound have to be studied. That is, we deal with the fashionable "hypersonic" speed range, and again suitable experimental conditions are just about reached now. One of the most interesting questions to be answered will be the possibility of supersonic turbulent fluctuations. It is quite possible that any velocity fluctuations in excess of sonic are rapidly damped such that "sonic velocity turbulence" in equilibrium with a shock wave field results. Münch's work on the Orion Nebula to a certain extent bears out this speculation.

The combination of high temperature and high velocity gases may make it even possible to study the partition of energy between turbulence, pressure waves, and magnetic-field fluctuations. This problem looks to me still very difficult. Experiments seemed impossible a few years ago. Today, I believe they look barely possible.

Finally, we have seen a fascinating display of phenomena in Bostick's experiments. Here we deal with electromagnetically controlled entities of a low pressure plasma. These entities do look like galaxies. But at this stage, I would certainly hesitate to conclude that we have the means to study galaxies with a scale factor of $10^{-22}$ ! I am more inclined to look at these experiments as studies of typical - and very interesting - configurations of plasma motion somewhat like the study of isolated vortex rings in ordinary gas dynamics.

Summarizing, I feel that this meeting has been very fruitful for the aerodynamicists. Many new possibilities and problems have been suggested. I am specifically grateful to the astrophysicists who tried to reduce some of their problems to the simplest, typical models. Because from these, the outsider can more easily grasp a definite problem without ending up in confusion overwhelmed by the wealth of observational detail. In the diffusion of interest and understanding between astrophysicists and aerodynamicists, we have made very much progress in the course of these meetings.

H. C. VAN DE HULST, Leiden Observatory, Leiden, Netherlands: In presenting a summary, I adopt the standpoint of an astronomer; this means that my classification is by the subjects in the universe, rather than by physical situations or problems. I shall go from small to large, and just review a few types of objects that we have talked about. As Burgers has already stated, one good point of progress in the course of these meetings has certainly been, that nowadays we talk less glibly about things that are present in a very small scale and again on a very big scale and then again very small. We have not jumped so much. We have more often used numerical values. This makes it slightly more tedious to listen, but it is really a progress in defining the problems more clearly.

I have roughly divided the topics into four classes:

(A) Planetary nebulae, nova shells, Crab Nebula, filamentary objects;

(B) Expanding complexes;

(C) Ordinary interstellar matter;

(D) Galactic-scale problems.

The Class (A) objects may be collected under the general name "shells," although there are quite different things in this group. Besides those listed, we have probably more that might be classed under (A) as objects that have been expelled by a star and have masses of the order of one stellar mass and not very much more. You remember that Minkowski divided the planetary nebulae into regular ones and the Chamber of Horrors. I wish to make clear that even these usually have a fair amount of symmetry, so that compared to them the objects under (B) and (C) are a forest of despair, I would say, because one really does not know exactly what he is talking about. As Liepmann has emphasized very strongly, the regular objects that can be well studied are, of course, the best things to start with in trying to understand them completely. Compared to those, the term Chamber of Horrors is really appropriate for the weird forms seen in other planetary nebulae.

Enormous progress has been made in the observations, both of the regular and of the irregular planetary nebulae, and also about the Crab Nebula and, as you have heard, about the more widely expanding shells. My hope is that within some years to come there may be a good bit of progress even with the rather peculiar objects, because further observations and further models, like those proposed in a discussion between Zanstra and Minkowski yesterday, may bring more clarity also into those objects. I have one question about these objects, which might be taken up later. I have not seen the evidence, nor heard a precise statement whether it is clear that these very thin filaments seen in the sky are really filaments or are shells. I thought it was implied that they were shells, but I should like to ask Minkowski to comment later whether they really are.

(B) Coming to the expanding complexes they have figured as the main agent in stirring up the interstellar medium. This was already discussed at great length in the 1953 Symposium. Also on these objects there has been good progress. As one example, I mention the fine 
study by Münch of the motions in the Orion Nebula. I should have liked to see more details of the work by Courtes with the interferometer, but that could not be presented. The theory of such expanding complexes with a hot star or a number of hot stars in the center has been reasonably well explored by Oort, Savedoff, $\mathrm{Kahn}$, and others, but there remain many questions. A spectacular question is this one: "Why are there three stars shooting out of the Orion region with enormous speeds; how can that ever happen?" If we wish to have a less spectacular question, it is to explain the details of the elephant trunks and bright rims that are seen in many complexes. This is a subject about which we have a fair amount of observational information now and about which there are beginning theories. To me it would be one of the most tantalizing problems to try to push ahead after the various remarks that have been made, both in favor of and against the RayleighTaylor instability at this conference. The experimental evidence that has been quoted and that might perhaps be relevant to this problem, should be discussed more precisely to see whether the circumstances in the experimental cases really allow us to draw astrophysical conclusions.

(C) Interstellar matter I should like to define in this summary as all parts of interstellar space, mainly inside a spiral arm, but perhaps also between, that have not recently been stirred up by the expanding complexes, but are just in some kind of irregular motion. Of course, we might hope that things will settle down to some extent after the first stir-up, so that, for instance, we can really tell from a photograph what the smallest size is below which there are no further details. I somehow had the impression from the Palomar Schmidt photographs that this smallest size might be a tenth of a parsec. Here lies a rather fruitful field of speculation, which is difficult because it does not refer to a precise model, yet which should be further explored in the coming years by people who have sufficient acquaintance with the other problems to use fair judgment in looking at the photographs.

Under the same subject, of course, falls the recurring question: "Are they really separate clouds or is there a field of hydrodynamical flow?" I would be tempted to answer: "Of course they are not clouds, of course there is a field of flow with pressure everywhere and density everywhere, but it is rather complicated. We are sure there are density differences of the order of 10 to 1 or more; we are sure there are large relative motions of the denser parts, and we are fairly certain that if we wish to have a crude estimate of the amount of dissipation we might, just for a moment, compare this field to a medium in which there are only clouds, which can "collide." This answer, however, definitely ignores a good part of the question. The role of the radiation and cooling mechanisms in determining the densities, pressures and temperatures has already been em- phasized. I should like to warn against oversimplification. It has been stated several times that the interstellar medium may be pictured as consisting of HII regions of that density and HI regions of this density, with pressure equilibrium, or not. All of such pictures are, of course, oversimplifications, but they may help us to feel our way.

(D) Finally, on the galactic scale, there are a host of problems, but many fall beyond the scope of this conference, because one of the main forces, the gravitational force, depends on the distribution of stars rather than of interstellar matter. However, a number of problems have been discussed. The forms of spiral arms is an old problem that has never been solved as yet. Why are there systems with and without a bar, just what are the motions in those spiral arms and bars, and what is their evolution? All these are problems for the future. Perhaps the observations of expansion near the center of our own galaxy are relevant in this respect, but we have to wait for further observations. To take a conservative point of view, I should like to look at the spiral arms just as regions of accidentally high density that have been drawn out by differential galactic rotation. Similarly for the magnetic field of spiral arms: regions in which the magnetic field was somewhat tangled up as usual, have been drawn out by differential rotation. This is the type of theory that Spitzer has proposed. From the evidence that has been presented until now, I see no reason for believing that the magnetic fields would have a more regular structure than this one.

The halo around the galactic system has been greatly discussed. This is the largest problem I shall talk about. Here the situation is that there are now two contradicting theories and both are supported by contradicting observations. This situation is, of course, very healthy, because it will probably be cleaned up within a year or two, but so far there is no reason to anticipate what will be the exact answer.

F. D. KAHN, Manchester, University, Manchester, England: Throughout the Symposium there has been a running controversy on just what are the correct figures on the rate of energy supply and dissipation. A central question is: How important is the question of the energy supply for the motion of the interstellar material? To see how the various numbers compare, let us contrast rate of dissipation at its most pessimistic value with the rate of output of radiant energy by the stars in our part of the galaxy. According to an old estimate of Eddington's, the radiation field in the galaxy is that of a blackbody at $10000^{\circ} \mathrm{K}$, diluted by a factor $10^{-14}$; this implies a radiant energy density of $3 \times 10^{-13} \mathrm{erg} / \mathrm{cm}^{3}$, and compares with a kinetic energy density in the turbulent motion of about $10^{-12} \mathrm{erg} / \mathrm{cm}^{3}$. That kinetic energy gets lost about once every ten million years. However, the radiant energy in the galaxy gets lost very much more quickly. If, for argument's sake, our part of the galaxy 
is taken to be 1500 light years thick, it will only be about 1000 years before the radiant energy escapes. It seems that the rate of loss of radiant energy is about 3000 times larger than the rate of loss of kinetic energy. Only a very small efficiency of conversion is needed to keep up the turbulent motion of the interstellar material. A typical mechanism of conversion being studied at present is that of the expansion of HII regions, but I do not see that we can prove this to be the only way in which stellar radiation can stir up interstellar material. There seems to be no very pressing need to reduce the rate of dissipation at the moment; no awkward fact forces us to do so.

While on this point of dissipation, I should like to make a point about the possible contributions due to turbulence on the largest, or galactic scale. Batchelor yesterday gave us reasons for believing that there would be no such turbulence with the observed variation of the circular velocity $v$ against radial distance $r$. Another reason, now possibly unnecessary, is this: as far as we know, the gas in the disk of the galaxy is concentrated into spiral arms, of which ours may be a typical one, and the $21-\mathrm{cm}$ observations indicate that such spiral arms have a more or less uniform angular velocity all the way along. They rotate almost like rigid bodies. There is an inner spiral arm rotating at a different speed, but there seems to be very little physical connection in the way of gas between that arm and ours. So it is hard to see how any velocity difference between the arms can stir up turbulence. We can conclude that no energy is degraded in this way, and I suppose two reasons are better than one for this conclusion.

Another important point was to understand the effect of a magnetic field on lightly ionized matter. There was less disagreement about the effect of a magnetic field on highly ionized matter. One reason is that the very interesting experiments of Bostick show what such a plasma will do in the laboratory. But I believe nobody makes experiments on lightly ionized matter which can be scaled up to apply to the galaxy. There is one observational astronomical result which may have something to do with the effects of magnetic fields on lightly ionized matter; some quite old interstellar spectra give a hint of a slight separation of the charged particles from the neutral particles. Thus, the study of the spectrum of the $\mathrm{Ca}^{+}$ion leads to a certain distribution of matter in interstellar space. The spectrum of the $\mathrm{Na}$ atom also gives a certain distribution of matter in interstellar space, and I believe that it is often found that the two cannot quite be reconciled. Perhaps the influence of the magnetic field, which is direct in the case of the $\mathrm{Ca}^{+}$ion and rather indirect in the case of the $\mathrm{Na}$ atom, may have something to do with it. One qualification should be made; the $\mathrm{Na}$ atom does not always stay neutral, but from time to time is ionized to become $\mathrm{Na}^{+}$. A comparison of the atomic and the ionic spectra of interstellar matter may help our attempts to understand the behavior of lightly ionized gases.

While speaking of the lightly ionized gas, I recall one puzzling point in Mestel's paper about the condensation of interstellar clouds which has to some extent been skated over, but which probably he could explain in one sentence, and that is this: He considers an interstellar cloud which collapses, becomes opaque, and in which the number of conducting particles is reduced. The current that was running there is then removed, along with the magnetic field. Will there be some self-induction effects in that case which should be considered?

Now a few words about the general theory of turbulence, supersonic turbulence, as the astronomer might like to have it developed. The general picture was explained roughly by Oort some years ago, and has hardly been changed at all. It is this: occasionally rather large lumps form in the interstellar gas. Inside these lumps condensation takes place, one or more bright stars will form, the gas is pushed away, and great pressure is exerted in the surrounding masses of gas. This, then, stirs up the turbulence. The turbulence takes its course and eventually another lump will form somewhere else. Anyone proposing a full theory of interstellar turbulence has to take into account the places where the next lot of stars are likely to form. All this is an extremely difficult problem. It must be remembered that a mass of gas at rest now has a good chance of being considerably stirred up during ten or fifty million years' time. Further, any theory must explain the connection between the following four quantities: (i) the dissipation rate, (ii) the Mach number, (iii) the scale, that is to say, the distance between the entities which have been called clouds but which, of course, are just regions of higher density and (iv) the value of $\left(\left\langle n^{2}\right\rangle_{\mathrm{Av}}\right) /(n)^{2}$, the degree of concentration of the material. There certainly will be a connection between quantities (i), (ii), and (iii), for the Mach number and the scale will determine the dissipation rate; and further the Mach number (ii) must be connected with the degree of concentration (iv). If a magnetic field is added as well, I will not venture to say what else needs to be considered.

As far as ionized regions are concerned, I think one change that we have seen at this Symposium is that there is now an observational reason for believing that HII regions are not uniform, but are really lumpy. I was very impressed with the evidence that Münch presented which showed how the density and state of motion can vary inside an HII region. Here is a challenge for theoreticians to try and explain the reasons. Münch's idea that the stirring up comes from outside is a very excellent one, and should certainly be explored by all of us.

The only other comment I wish to make is on the 
very interesting suggestion due to Shklovsky and mentioned by Pickelner about the radio source CygnusA. The idea is that here we observe a collision, not between two galaxies, but between two galactic halos and that this produces the radio source. The collision sets up shock waves which find their way to the far end of the two halos. This would explain the peculiar picture which Jennison and Das Gupta found for this object. I am sure that lots of people will be wanting to work out this suggestion, but wonder how many will succeed.

L. SPITZER, JR.: The foregoing represent impressions on various aspects of the Symposium as a whole. We have now two summarizing contributions on two specific subjects-cooling mechanisms and the fraction of radiative energetic output available for conversion to mechanical energy.

M. J. Seaton, University College, University of London, London, England: The various cooling mechanisms result from collisional excitation of some sort followed by radiation.

$$
\begin{aligned}
A_{i}+B & \rightarrow A_{j}+B \\
A_{j} & \rightarrow A_{i}+h \nu .
\end{aligned}
$$

The energy loss is

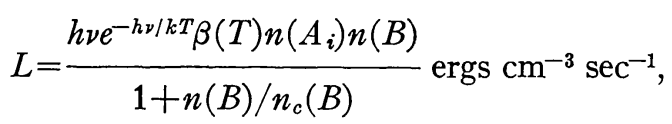

where $n_{c}(B)=a_{j i} / q_{j i}\left(a_{j i}\right.$ being the radiative transition probability and $q_{j i}$ the rate coefficient for collisional de-excitation) and $\beta(T)=\left(\omega_{j} / \omega_{i}\right) q_{j i}$. If $n\left(A_{i}\right)$ and $n(B)$ are both assumed proportional to the total particle density $n, L$ will be proportional to $n^{2}$ for $n(B) \ll n_{c}(B)$ and to $n$ for $n(B) \gg n_{c}(B)$.

1. Ionized Nebulae: Cooling is mainly due to electron excitation of forbidden lines, for which $(h \nu / k) \sim 3 \times 10^{4}$ ${ }^{\circ} \mathrm{K}$. Equating energy loss to energy gain by photoionization, the calculated electron temperature is insensitive to the color temperature of the ionizing radiation and is of order $10^{4}{ }^{\circ} \mathrm{K}$. The ionization equilibrium may depend on density in such a way that the cooling rate is much greater in the denser regions (Zanstra mechanism). The critical electron density $n_{c}(e)$ is of order $10^{5}-10^{6} \mathrm{~cm}^{-3}$.

2. Neutral Hydrogen Clouds: We adopt the usual model in which clouds with density $n(\mathrm{H})=10 \mathrm{~cm}^{-3}$ occupy $10 \%$ of the total volume. The mean energy loss is $\bar{L}=0.1 L$, where $L$ is calculated for the clouds

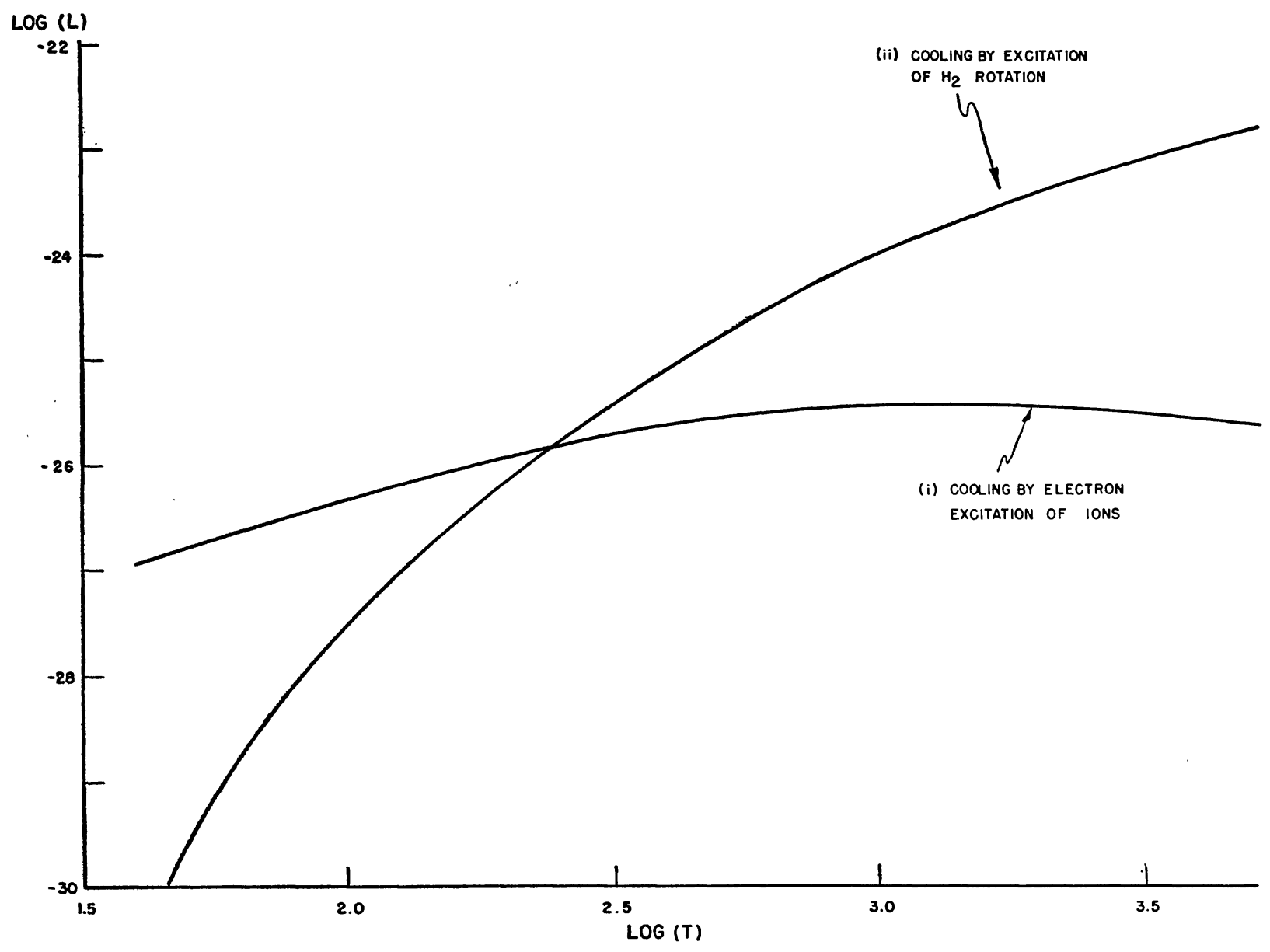

FIG. 1. Energy loss $L$ calculated for $n(\mathrm{H})=10 \mathrm{~cm}^{-3}$. (i) Electron excitation of ions assuming normal abundances; (ii) excitation of $\mathrm{H}_{2}$ rotation assuming $n\left(\mathrm{H}_{2}\right)=10^{-2} \mathrm{~cm}^{-3}$. 
Two mechanisms may occur: (i) electron-ion collisions and (ii) excitation of $\mathrm{H}_{2}$ rotation.

(i) With abundances assumed similar to typical stellar abundances, one may calculate the cooling resulting from excitation of fine structure levels in $\mathrm{C}^{+}\left(h \nu / k=92^{\circ} \mathrm{K}\right), \quad \mathrm{Si}^{+}\left(h \nu / k=413^{\circ} \mathrm{K}\right)$ and $\mathrm{Fe}^{+}(h \nu / k$ $=554$ and $\left.961^{\circ} \mathrm{K}\right)$. Results are given in Fig. 1. In the clouds $n(e) \simeq 2 \times 10^{-3}$ compared with $n_{c}(e) \sim 10^{4} \mathrm{~cm}^{-3}$. The assumed abundances are unlikely to be in serious error for the over-all composition of interstellar matter but the possibility may be considered that most of the $\mathrm{C}, \mathrm{Si}$, and $\mathrm{Fe}$ is condensed in grains. This would imply a large number of grains on which $\mathrm{H}_{2}$ molecules would form.

(ii) For $n(\mathrm{H})=10$ the calculations of Kahn (Liège, 1954) suggest that $n\left(\mathrm{H}_{2}\right)$ is at least $10^{-2} \mathrm{~cm}^{-3}$. The resulting energy loss $L$ due to excitation of $\mathrm{H}_{2}$ rotation is shown in Fig. 1. For $T$ of order $100^{\circ} \mathrm{K} . n_{c}(\mathrm{H}) \simeq 3$ $\mathrm{cm}^{-3}$. For excitation of the first $\mathrm{H}_{2}$ rotational level, $(h \nu / k)=520^{\circ} \mathrm{K}$.

The dynamical model in which the gas is heated to $3000^{\circ} \mathrm{K}$ once in $10^{7}$ years corresponds to a rate of thermal energy gain $\bar{G}=2 \times 10^{-27} \mathrm{erg} \mathrm{cm}^{-3} \mathrm{sec}^{-1}$. Either one of the two cooling curves are consistent with this model and with a harmonic mean temperature close to $125^{\circ} \mathrm{K}$. If $T$ is assumed constant at $125^{\circ} \mathrm{K}$, the cooling rate $\bar{L}$ is $6 \times 10^{-28} \mathrm{erg} \mathrm{cm}^{-3} \mathrm{sec}^{-1}$ for electron-ion cooling and $1 \times 10^{-28} \mathrm{erg} \mathrm{cm}^{-3} \mathrm{sec}^{-1}$ for $\mathrm{H}_{2}$ cooling.

3. Time Scale for Cooling: As has been emphasized by Pickelner, the time required for cooling of an ionized gas will be short compared with the time required for recombination. This is of importance when considering what happens when the source of ionization is removed.

J. C. PECKer, Observatorie d'Paris, Meudon, France, AND E. Schatzman, Institut d'Astrophysique, Paris, France: An important point, in the discussion of the first two days of the meeting, has been the estimation of that part of the energy output of all stars which is transformed into mechanical energy.

Van de Hulst recalled that Oort's estimate of that quantity (based on the observed big gaseous complexes) is rather a lower limit. On the contrary, the figure given by Schlüter at the second of these symposia was based on an estimate of the total energy output in $\mathrm{O}$ and B stars, and on counts of those stars.

Two possible causes of error may affect the estimate of the total energy output. First, the use of Kuiper's value for the bolometric correction leads to an underestimate of the effective temperature. The change in the total flux is of the order of $10 \%{ }^{1}$ Second, more important, effect comes from the fact that, on the violet side of the Lyman discontinuity, the actual flux is much smaller than the flux one would have if the stars were radiating as a blackbody at the effective tempera-

1 A. B. Underhill and J. K. McDonald, Astrophys. J. 115, 577 (1952).
TABLE I.

\begin{tabular}{clcccc}
\hline \hline Sp. Type & \multicolumn{1}{c}{ Author } & $T_{\text {eff }}$ & $T_{0}$ & $Y_{m} / Y_{\text {eff }}$ & $Y_{m} / Y_{0}$ \\
\hline O5 & A. B. Underhill, & 44600 & 30000 & 0.60 & 3.1 \\
B1.5 & $\begin{array}{l}\text { J. C. Pecker, } \\
1050\end{array}$ & 26400 & 17300 & 0.08 & 1.0 \\
\hline \hline
\end{tabular}

ture, $T_{\text {eff }}$ as usually assumed in the former analyses. Consider how the use of a detailed theory of stellar atmospheres can modify these data.

Some models of O.B stars are available, and we know the corresponding ultraviolet fluxes. From the published O5 model of Underhill ${ }^{2}$ and B1 model of Pecker, ${ }^{3}$ we have computed (1950) the total energy output in the ultraviolet, which output is proportional to the quantity

$$
Y_{m}=\int_{\nu_{1}}^{\infty} \frac{F_{\nu}}{h \nu} d \nu
$$

and have compared that quantity to the quantities

$$
Y_{\text {eff }}=\int_{\nu_{1}}^{\infty} \frac{B_{\nu}\left(T_{\text {eff }}\right)}{h \nu} d \nu \text { and } \quad Y_{0}=\int_{\nu_{1}}^{\infty} \frac{B_{\nu}\left(T_{0}\right)}{h \nu} d \nu .
$$

$\nu_{1}$ is $\nu$ at the Lyman limit. Table I recalls the principal characteristics of these models, and gives the ratios $Y_{m} / Y_{\text {eff }}, Y_{m} / Y_{0}$. In the case of the $\mathrm{B} 1$ star, the result is that the classical value of the ultraviolet energy output is overestimated by a factor 12.5 . In the case of the $\mathrm{O} 5 \mathrm{star}$, the effect is only a factor 1.7.

We may note that these values are rather uncertain, being quite sensitive to the computation of the model. In a B2 model computed by McDonald, ${ }^{4}$ the correction factor is much bigger than in the B1.5 model of the table. Moreover, the possible presence of departure from local thermodynamical equilibrium in the outer layers (existence of a chromosphere) makes the problem still more difficult.

If one wants to use a single temperature in the determination of the ultraviolet energy output, it is better to use the "surface" temperature, $T_{0}$, than the effective temperature. The results with $T_{0}$ are included in the table. For the central stars of planetary nebulae, Zanstra's method provides a temperature which is even more reliable.

Naturally, it should be necessary to take full account of the statistics of the different spectral types. The total effect could be to decrease Schlüter's value by about one order of magnitude.

One could summarize the result by stating that an important part of the ultraviolet radiation, formerly

${ }^{2}$ A. B. Underhill, Publs. Dominion Astrophys. Observatory 8, 357 (1950).

${ }^{3}$ J. C. Pecker, Ann. astrophys. 13, 433 (1950).

${ }^{4}$ J. D. McDonald, Publs. Dominion Astrophys. Observatory 9, 269 (1953). 
considered as used to produce ionization of hydrogen, and mechanical energy, is indeed used to maintain the radiative equilibrium in the outer parts of the stellar photospheres.

L. SPITZER, JR. : I would like to use the inalienable right of the chairman to summarize briefly my impressions as to where we are in this broad field of the motions of a gas of cosmic dimensions. To begin with, I suggest a breakdown into two headings, kinematics and dynamics. It seems to be generally true in astrophysics -as for example, in close binary stars, in stellar atmospheres and the outer layers of the sun, in nebulae, and in interstellar matter generally - that the subject of kinematics, the observational exploration of the velocity and density field, is a subject in which we can hope to make very significant progress, and in which we do have a very real understanding. In contrast, however, the subject of dynamics, the physical explanation of how and why the observed motions arise, is a field concerning which we know relatively little in almost any branch of astronomy. We have made progress in the last three symposia on the kinematics of the interstellar material, but I am persuaded that the progress we have made on dynamics is really rather slight. We have progressed in that we have increasingly defined the problem, we have thrown out certain hypotheses, we know what problems there are to be investigated, but our understanding of the dynamics of the interstellar material remains very tentative.

If I might simplify van de Hulst's table, there are two general areas which have been investigated under kinematics. First, there are isolated nebulae, systems that one can look at as single structures without considering the galaxy as a whole. Here we have a great deal of information. There are the beautiful observations from the $\mathrm{Mt}$. Wilson-Palomar group on planetaries and on the Orion Nebula which begin to shape up into a consistent picture. We can hope that we really begin to understand what the velocity field looks like in some of these systems. In the next few years, we will perhaps know even more than we do now. Generally, the 21-cm observations from the expanding HII regions have also led to a very consistent picture of the velocities in these isolated nebulae.

The second area where extensive kinematical data have been obtained is the general field of interstellar gas. While the picture is not so complete as for isolated nebulae, there are a number of rather firm observational results. We have a general idea, both from the calcium and sodium absorption-line observations, and from the $21 \mathrm{~cm}$ data, of the general range of velocity in the interstellar gas. We have a general picture from star counts, photographs, and other data as to the irregular distribution of the material of interstellar space. What is lacking, as van de Hulst pointed out, is the fusion of the velocity data and the density data. At present, we cannot really state in detail what the densities and velocities at a particular point in the interstellar gas are.

In fact it is quite likely that the situation is intrinsically very complicated, and it is not at all obvious how one should try to summarize this information. If one looks out at a cloud going by on this hot summer afternoon, and asks what is the density and velocity field in the lower atmospheres above Cambridge at this moment, it is a little difficult to know just how that question can most profitably be answered. One might perhaps hope actually to make a three-dimensional diagram and give the density and velocity at every point. This is clearly the complete way of answering the question, but I hope that we do not have to get involved in that detail. If one tries to give a statistical picture of the clouds that go by, it is not at all obvious what statistical framework is appropriate, because there are little filaments in the big clouds, and there are groups of big clouds. In the case of the clouds over Cambridge it is obvious just by looking at them what the actual situation is, in general terms, but it is not at all obvious how extensive data on the subject might best be summarized.

It is clear that the situation is rather complicated for the interstellar gas. It is not certain that we have asked the best questions, but it is certain that in particular areas we do have information, and we have a general conception of what the problems are.

When we come to dynamics, perhaps our present state of ignorance is best summarized by following Burgers, and listing as milestones the three separate symposia we have had on this subject: Paris in 1949, Cambridge, England, in 1953, and Cambridge, Massachusetts in 1957. When I look at these three dates, I am reminded of the poem by Browning on the philosopher Cleon, who, as you remember, had written three books on the soul, "proving absurd all written hitherto and putting us to ignorance again." If I may simplify very much, each of these symposia may be identified with a particular dynamical theme. While many dynamical subjects have been discussed at each symposium, one may select one topic which was particularly emphasized at each meeting.

At the first Symposium, perhaps the chief dynamical topic was the theory of isotropic turbulence. Observations were cited showing that isotropic turbulence seemed to be present. There were theories indicating that isotropic turbulence might perhaps arise. At this meeting, the Mt. Wilson group have thrown out the observations in the Orion Nebulae which indicated isotropic turbulence, the $21 \mathrm{~cm}$ observations have shown that large-scale turbulence does not exist, and now Batchelor tells us that turbulence would not really be expected to arise anyway from a rotating mass of gas. So one result of our deliberations is that, in 1957, we can throw out the theories of 1949 and conclude that isotropic turbulence is probably not very relevant, at least on a large scale. 
The theme of the second Symposium, in 1953, can perhaps be listed as the thermal expansion of gas around newly born early-type stars. Since I have a certain vested interest in this field, I will not go so far as to say that in this symposium we have thrown out this mechanism of expansion around $\mathrm{O}$ stars, but certainly the relevance of this effect has been called into question; there seems to be some question as to whether the available energy is great enough for the dissipation that we believe may be present. My own view is that there is sufficient energy by a small margin. I shall present my views on this matter in the more general discussion, but I think we do not at this Symposium get the feeling of clear confidence that this mechanism might be the answer to all our dynamical prayers that one receives from the proceedings of the 1953 Symposium.

The chief theme of the present conference seems to be hydromagnetics. Almost all the dynamical papers that have been discussed today have mentioned the magnetic field. There is a marked contrast between hydromagnetics and the quite different topic of thermal processes in a gas which Seaton has just summarized. I have not included thermal processes in my brief outline, because such effects are really outside dynamics, but in discussing thermal processes, I believe we are on fairly firm ground. When we come to discussing hydromagnetics, however, we compound the difficulties of dynamics. In the subject of pure dynamics without magnetic fields, quite a bit of firm information is available. In the subject of hydromagnetics, there is very little that is definitely known that can be applied to interstellar gases. Solutions are known of the hydromagnetic equations for certain simplified cases. These simplified cases are probably not too relevant for the interstellar gas, and we have the uneasy feeling looking at hydromagnetics that an ionized gas may be capable of types of motions that at the present time we do not even suspect. When we become involved with hydromagnetics, we are getting into a field which is only in its infancy and much more theoretical and experimental work is going to be required before we can hope to apply the necessarily simple concepts of theory to the complicated phenomena that are unquestionably going on in interstellar space.

In general, when we consider the dynamics of cosmic motions, we are really undertaking a very ambitious task. It is a little as though we have an observatory on the moon and are investigating weather on the earth under rather restrictive conditions. Let us suppose that we are confined not to looking at the weather as it develops but to making measurements during one hour only; we can take all the observations we want during that hour, but we cannot look before or after. From the spectroscopic observations of clouds on the earth, taken essentially at one moment of time, we would then be required to construct a theory of dynamic meteorology! Actually this hypothetical problem, difficult though it seems, is probably much simpler than the problem we are trying to tackle, because the thermal processes on the earth are probably somewhat simpler than the processes that take place in interstellar space. Moreover, in terrestial weather, you can probably neglect the magnetic field for most applications. In interstellar space, we have an additional vector function of position, whose influence on the motions may be very profound and very great.

We turn now to the general discussion. Let me initiate it by commenting on the Pecker-Schatzman point and review the energy input from $\mathrm{O}$ stars. The following figures give the total energy radiated from $\mathrm{O}$ and $\mathrm{B}$ stars beyond the Lyman limit, obtained in an earlier computation of mine. The units are ergs per $\mathrm{cm}^{3}$ per sec in the galactic plane, in the solar neighborhood:

$\begin{array}{llllllll}\text { Spectral class: } & 05 & 6 & 7 & 8 & 9 & \text { BO } & 1\end{array}$ $\begin{array}{llllllll}\text { Energy }\left(\mathrm{erg} \mathrm{cm}^{-3}\right): & 1.1 \times 10^{-25} & 1.0 & 0.41 & 0.34 & 0.24 & 0.05 & 0.03\end{array}$

These data were derived from the blackbody formula and from statistics on the number of stars of various spectral classes per cubic parsec in the vicinity of the sun. If one adds all contributions, one gets a total power of about $3 \times 10^{-25} \mathrm{erg} / \mathrm{cm}^{3} \mathrm{sec}$ radiated beyond the Lyman limit. According to the calculations just presented by Kahn and by Pecker and Schatzman, the blackbody formula overestimates the energy by a factor between 2 to 10 . Since most of the energy comes from the very early stars, we can reduce the computed power by a factor of 3, according to the Schatzman-Pecker results and so end up with about $10^{-25} \mathrm{erg} / \mathrm{cm}^{3} \mathrm{sec}$, with an error not likely to exceed a factor of two.

We next ask how much of this energy can be converted into dynamical or kinetic energy. The efficiency factor, even in the most ideal case, cannot exceed 0.1 , because the photons are very energetic, 14 to $15 \mathrm{ev}$. These photons are absorbed; $13.5 \mathrm{ev}$ goes into potential energy, leaving only a volt or two that goes into heating the gas. So the energy available for heating the HII regions is roughly $10^{-26} \mathrm{erg} / \mathrm{cm}^{3} \mathrm{sec}$. The energy required for the dissipation computed for cloud collisions is about $10^{-27} \mathrm{erg} / \mathrm{cm}^{3} \mathrm{sec}$; so if we wish to assume that the energy dissipated is provided by this basic mechanism, we must assume that an appreciable fraction, at least $10 \%$, of the thermal energy absorbed in HII regions goes into actual dynamical energy. Now it is not at all certain that so large a fraction of the thermal energy of HII regions will, in fact, go into kinetic energy, but there is some margin of safety, and it is on this basis that I stated my own conclusion a little earlier that there probably was enough energy from the $\mathrm{O}$ stars to explain the dissipation.

M. P. SAVEDOFF, University of Rochester, Rochester, New York: Note that this energy has to go into neutral gas. I should say the neutral gas clouds have a longer lifetime as a distinct unit than clouds in the ionized gas. 
If you put all your energy into just expanding ionized gas, you obtain motion, but this is short lived, and is not useful to us.

T. K. Menon, Harvard College Observatory, Cambridge, Massachusetts: I think van de Hulst passed over one point very quickly. As a result I do not know whether or not the wrong impression has been created. He mentioned that three stars in the Orion region originated in the Orion Nebula or are accelerated away from the Orion Nebula. I do not think it can be just taken for granted that that is the case, because the center of expansion of these three stars and the center of expansion from $21 \mathrm{~cm}$ observations are not coincident with the Orion Nebula at all. Moreover, as Spitzer and Oort showed, the energy necessary for the acceleration of these three stars is not available from the early type stars in this region by any of the known mechanisms. Hence, I think one has to consider the problem from a different angle.

L. Mestel, Cambridge, England: I should like to reply to a query raised in Kahn's summary. Selfinduction is taken care of by Faraday-Neumann equation $\boldsymbol{\nabla} \times \mathbf{E}=-\partial \mathbf{H} / c \partial t$. The equation for the drift of the electrons relative to the ions is approximately $\mathbf{E}+\left(\mathbf{V}_{i} \times \mathbf{H} / c\right)=0$, where $\mathbf{V}_{i}$ is the plasma velocity; the neglected terms, such as the ohmic field $\mathbf{j} / \sigma$, are usually small in cosmical applications. Combining these two equations we have $(\partial \mathbf{H} / \partial t)=\boldsymbol{\nabla} \times\left(\mathbf{V}_{i} \times \mathbf{H}\right)$ giving the time variation of the field. This is identical with the familiar equation of hydromagnetics, except that $\mathbf{V}_{i}$ replaces $\mathbf{V}$, the velocity of the gas as a whole. We may immediately conclude that the magnetic field is tied to the plasma, instead of to the gas as a whole. The variation of the magnetic field is, therefore, determined by the forces acting on the plasma, principally the magnetic force $(\mathbf{j} \times \mathbf{H} / c)$, and the friction between the ions and the neutral atoms. In the paper presented here, I dealt with the problem of a contracting protostar with its internal magnetic field frozen in; i.e., I assumed explicitly that the plasma density is high enough for coupling between plasma and neutral gas to be strong, so that $\mathbf{V}_{i} \approx \mathbf{V}$. This insures that the field moves effectively with the gas as a whole. On the other hand, Spitzer and I have shown that if the plasma density drops to about $1 / 100$ th its normal value, the coupling between the two components is small enough for the neutral matter to flow without dragging the plasma and the magnetic field tied to it. In both problems the forces acting on the plasma determine its motion, while self-induction, operating in a region of large dimensions, ensures that the field follows the motion of the plasma.

H. D. GREYBER, General Electric Research Laboratory, Schenectady, New York: In connection with turbulence and its origin, I would remark on a general instability which has been known for 50 or 60 years as Helmholtz instability. If you have two contiguous fluids, one with velocity $V_{1}$ and the other with a parallel velocity $V_{2}$, and if a perturbation developed on the interface, it is unstable and will grow and generate turbulence. Of course, this result holds for the sharp interface, but I feel that instability would result also for a gradual change of velocities. The analysis has been done for the hydromagnetic case with a plasma, and you get this instability again. These effects may be of interest to produce turbulence under a system of differential rotation.

J. M. BURGERS: Greyber notes that instability appears when there is a discontinuity in the velocity, and suggests that something similar may occur for a gradual change of the velocity. But as soon as one starts to consider a continuous distribution of velocity, one runs into the general problem of hydrodynamic stability with all its difficulties. In the case of incompressible Poiseuille flow, or of boundary flow, highly complicated numerical calculations have been necessary in order to find out where the limit occurs between stability and instability. Hence, one must expect that the problem must be even much more difficult when we consider a gaseous mass, floating in space, bounded not by rigid surfaces but gradually tapering off in density under the influence of a gravitational field, even if we leave out the magnetic forces. Nobody, therefore, can say whether such a simple criterion will be decisive or not.

H. D. GREYBER: You are quite right. This has not been done, but the special case of Taylor instability of a horizontal plane interface whose density changes smoothly from one constant value on one side to another constant value on the other side has been treated. If a similar case including Helmholtz instability with the velocity changing gradually could be analyzed, then perhaps some light would be shed on the more difficult general problem described by Burgers.

G. K. BATCheloR, Trinity College, Cambridge, England: It certainly seems reasonable that where there are large amounts of kinetic energy, there will almost certainly be a turbulent motion, as a result of some instability, with details dependent upon the particular case concerned. But, I emphasize again, for instability to occur there should not be present any strong stabilizing field of force. The particular source of turbulence referred to earlier in this Symposium is galactic rotation, and there the simple Rayleigh criterion shows that the galactic rotation does indeed have an associated stabilizing field of centrifugal force. Inasmuch as the galactic rotation is two dimensional, with axial symmetry, there is no reason to believe large scale turbulence can develop from it. But, of course, any smaller scale motions perhaps arising from variation of the rotational velocity with respect to distance 
normal to the galactic plane-such motions are likely to generate turbulence, as remarked.

H. LIEPMANN : I would like to say just a word of caution in identifying instability with turbulence. For example, the Taylor vortices or the instability modes produced by gravitation, are not turbulence, and large scale instability is neither necessary nor sufficient to get turbulence, in some cases. These instability modes are large-scale organized motions. The typical case is flow between concentric cylinders obtained by rotating one clyinder with respect to the other. Here one obtains these large cells as Taylor first showed, but they in themselves are not turbulence, and they can persist for an infinite time without developing turbulence.

L. BIERMANN, Max Planck Institut Für Physik, Göttingen, Germany: If it is agreed that the main supply of energy comes from hot stars, could one not say that to a certain degree the uneven distribution and the short life of these hot stars, simulates in a way a sort of turbulence? That is to say, if they are distributed suffciently short lived, so that on the galactic scale here or there a star is shining and again disappearing, we might have a picture which resembles turbulence without being turbulence in the strict sense of the aerodynamicists.

L. SPITZER, JR. : This is certainly a most interesting suggestion, especially since the rate of star formation may presumably be related in some way to the velocity field at a previous point. Perhaps one could analyze the interstellar velocity field by essentially introducing an energy source which depended on the conditions of velocity and pressure at a particular point.

J. M. BURGERS : I believe this suggestion already came up at the Symposium of 1953. It is a very important point of view: compressible turbulence may well be due to the presence of random sources of energy, while, on the other hand, the appearance of such sources in some way may be linked with the density distribution. I have hinted in this direction in my summary of this morning. We thus may have a cycle of relations which could lead to a form of instability and of turbulence quite different from that presented by the usual picture which starts with cases in which shear flow is the important consideration.

R. N. THomas, National Bureau of Standards, Boulder, Colorado: I would just like to come back to the remarks that I made a long time ago on these things. Biermann's is certainly a very interesting suggestion, but if one comes down to the actual practical problem of describing it; for example, in a situation, where I have a uniform expansion of material out from each of several stars, then locally I have nothing resembling turbulence. A discussion of instability leading to turbulence, Reynolds numbers, a spectrum function derived from turbulence arguments-these are meaningless in this situation. It is only if I shrink the scale and look, with low resolution, that I see a random set of motions. But dissipation processes are local processes, and one must then discuss the local equations of motion. One should describe this as a systematic set of motions intersecting, and interacting, not as a system of turbulence on a local scale. The important thing is the interaction. The one thing in astronomy one wants to do is to use clear-cut physical concepts; we have had enough trouble in the last 25 years with a nondefined and misapplied turbulence concept. Almost everyone jumped on the Kolmogoroff turbulence bandwagon in the postwar years, and got nowhere because it was inapplicable. The approach ignored the interactions of velocities which are apparently, from observations, supersonic, hence highly compressible. If the situation is systematic motions, let us talk about symmetric motions, and not introduce a fictitious "turbulence" to whose interpretation we misapply legitimate aerodynamic turbulence concepts.

S. B. PiCKelneR, Crimean Astrophysical Observatory, Simeis, U.S.S.R.: I should like to direct attention to several questions which now are not clear. In the center of our galaxy, there are fast movements and the radio observations show that the velocity of these movements increases towards the center. It is necessary to find concrete mechanisms for the origin of the velocity in the center. It may be contraction of the whole galaxy, and it is possible that the gravitational energy of the galaxy may transform into kinetic energy. If the galaxy is contracting at a rate of only $1 \mathrm{~km} / \mathrm{sec}$, this is enough to support the dissipation of about $10^{-27} \mathrm{erg} / \mathrm{cm}^{3} \mathrm{sec}$ over the whole volume of the halo. A second problem, emphasized by Shklowsky, is the quasi-equilibrium of the energy between the relativistic particles and the magnetic or the kinetic energy. In the Crab Nebula, as in the halo, the relativistic particle energy is the same as the magnetic energy. This fact is not trivial.

L. SPITZER, JR.: Is your estimate based on the total mass of the stars in the galaxy?

S. B. PICKELNER: Yes, on the total mass of the stars.

L. SPITZER, JR. : If one assumes that the mass of the gas is a small fraction of the mass of the stars, would you not have to increase your figure of $10^{-27} \mathrm{erg} / \mathrm{cm}^{3}$ sec, because the stars probably are not coupled to the gas in any very effective way?

S. B. PICKELNER: The contraction of the stars of the galaxy may change the gravitational field in the galaxy. The star's movements may be accelerated this way. There are no clear mechanisms of the transformation of this energy into kinetic energy of the gas, but 
it is possible that the movements of the star streams might introduce turbulence in the gas. I pay attention only to the fact that the energy dissipation in our galaxy for several billion years is of the same order as the total gravitational energy of the galaxy as a whole. There are not so many energy sources in our galaxyonly gravitational energy and nuclear energy in the stars-and we have to discuss all the possible mechanisms. It is important that the fast movements are in the central region of the galaxy, where there are no hot stars.

E. SchatZMAN : I would like to emphasize one aspect of the cooling problem which has been overlooked. There are two parts to this problem-getting the "coolant" and effecting the cooling. The last has been covered well by Seaton. Consider the former, and write two equations; the first gives the change in ionization equilibrium:

$$
\frac{\partial n_{H}}{\partial t}=g n_{e} n_{p}-K J n_{H},
$$

where $J$ is the mean intensity of radiation. The second is the transfer equation for radiation:

$$
\frac{\partial I}{\partial \tau}=-I+\varpi J+\varpi S,
$$

where $\varpi<1$ and $S$ is the source function. As long as there is enough radiation to keep the matter ionized, even if the level of ionization is low, it is extremely difficult to have the matter cooling down, and it is only when $J$ has been reduced considerably that it would start cooling down according to the efficient mechanism which has been described by Seaton. So the question is, how can $J$ become small? To make $J$ small you need to introduce some neutral hydrogen which would reduce the intensity. And that can be achieved only by some kind of instability in the HII region. I must say that I do not see any other way of having it cooling down.

F. D. KAHN : I have a question which concerns the rate of cooling in interstellar space. Seaton has drawn a diagram of the variation of temperature with time in a particular cloud, something like that shown in Fig. 2. The cloud is heated, cools, is heated again, cools, and so on. The $21-\mathrm{cm}$ line may be used to measure the harmonic mean of the temperature between one

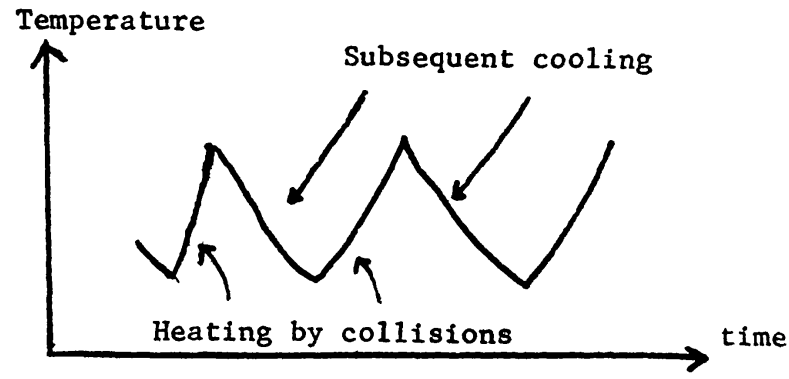

Fig. 2. collision and the next. But the harmonic mean is extremely weak in determining the peak temperatures. One can easily consider a model where the peaks are very much lower and still get much the same harmonic mean. But lower peak temperatures mean a smaller rate of dissipation of energy. Can somebody think of some way of measuring, not average of $1 / T$, but the average of some other power of $T$ to get a check on this point?

L. SPITZER, JR. : Two types of useful measurements could, in principle, be made. One possibility is to observe the radiation from the interstellar material in the far infrared, in the so-called dark octaves between 10 and $100 \mu$ or perhaps even $1000 \mu$. This is the wavelength region where the cooling radiation for the interstellar clouds is located. The atmosphere is relatively opaque at such wavelengths, but observations could be made from a balloon at some 20 miles altitude. To measure the continuous radiation from the Milky Way in the region 20 to $50 \mu$ would be a very interesting observation, because it would determine directly the amount of radiation absorbed by the interstellar grains. If the interstellar grains have an albedo of perhaps $\frac{1}{2}$ they should re-radiate in the infrared and energy about equal to that which they scatter, or half of the total radiation extinguished. From this, one finds immediately that the total radiation in the far infrared should have an energy about equal to the total energy in starlight, which would be a quite appreciable amount of radiation. I think looking in the far infrared holds open, in principle, the possibility of considerably extending our horizons in the physical analysis of the kinematics of interstellar matter.

Another way of extending our information in this field is to try and extend the observations of the interstellar absorption lines. I believe that this type of work has gone about as far as it can with the existing techniques, but with photoelectric methods one can go very much farther in measuring very faint lines. For example, by looking at the interstellar lines of neutral calcium which are too weak to be seen on photographs of stellar spectra, one can hope to find much more detailed information on the electron density in interstellar space. Another possibility is to look at the neutral calcium intensity in the high velocity clouds, to test Kahn's assumption that the reason for the abundance of sodium is that the neutral sodium atoms do not get carried along with the hydromagnetically moving clouds. If this assumption is correct, the density of neutral calcium should be anomalously low in a high velocity cloud. There are many things that one can do with photoelectric observations of interstellar absorption lines.

H. C. VAN DE HULST : I just want to remark that there are still a number of interstellar lines, or bands, 
that have defied all attempts to identify them with known molecules or substances. If we knew what causes these lines, perhaps we could go further in our speculations.

F. DRAKE, Harvard College Observatory, Cambridge, Massachusetts: With regard to the radiation in the infrared in the range of about 10 to $100 \mu$, Gold (in private discussions) has noted the great significance of measuring this radiation, as Spitzer has just pointed out, and the possibilities of observing it have been looked into in considerable detail here at Harvard. There is a window in the atmosphere at about $12 \mu$ which would be suitable for the study of this radiation, and there is also a very sensitive infrared detector that exists for these wavelengths, so that it is possible to observe this radiation. However, there is a difficulty in that one is forced to observe low intensities of brightness temperature, using a radio astronomy term, of about, we would expect, 15 degrees or so, against a sky background of approximately 300 degrees, because our atmosphere is opaque at the nearby wavelengths and therefore would introduce a noise level equivalent to 300 degrees. One is thus faced with the instrumental problem of obtaining filters which are cooled to temperatures near absolute zero, but which transmit only in the region around $12 \mu$, and this problem has to be solved before these observations are feasible.

L. SPITZER, JR.: What is the detector that you have in mind; and secondly, have you looked into the opacities of the atmosphere at a height of 20 miles?

F. DRAKE: There are several detectors: immersed thermistor bolometers, a doped germanium cell, an indium sulfide cell, and some others which are still being developed. The height of 20 miles probably would offer no problem. As a matter of practicality, an attempt to develop a system that would be effective from a high mountain seems desirable; from an earth satellite observations are quite possible now.

L. SPITZER, JR.: Twenty miles? That is not satellite altitude. Above a height of 20 miles there is only $2 \%$ of the air and very little water vapor. It is not at all difficult to send equipment up on a balloon to such altitudes.

F. DRAKE : Offhand this would seem quite practical. I would have to compute it. It would appear that at that altitude the observational problem would be greatly reduced and observations should be quite easily obtained.

R. W. STEWART, University of British Columbia, Vancouver, B. C., Canada: It has been suggested several times that HI clouds at temperatures around $100^{\circ} \mathrm{K}$ are surrounded by HII regions of temperatures 100 times that and the pressures are about equal. Equal pressures means that we have the same momentum transfer. With velocities in the ratio about 10 to 1 , we have one molecule going into the cloud and 10 coming out, which means that the HI cloud diffuses outward at a speed approximately equal to $1 \mathrm{~km} / \mathrm{sec}$. After a while, there would not be any clouds, and the only way you can overcome this is to imagine a mass velocity into the HI cloud at about the same rate as the diffusion rate, so that the net position of the boundary remains fixed. But to maintain such a motion, we would require a good deal higher pressure in the HII region, than we have in the HI region.

L. SPITZER, JR. : I think the problem is essentially that of a container with a hot gas at the top and cold gas at the bottom; what is the rate of diffusion between these gases? The diffusion rate depends upon the mean free path, and is small if the mean free path is much less than the container dimensions. In an interstellar cloud the mean free path for ions is only a few astronomical units. For the neutral particles, it is somewhat longer, but is still considerably less than the cloud radius, which may be taken as about $10 \mathrm{pc}$. Certainly this raises an interesting point, but I have the impression that the mean free path is sufficiently short compared with the cloud dimensions, so that is probably not serious.

J. M. BURGERS : I think the problem is not much more difficult than one which was discussed 8 years ago at the Paris meeting, when we had in mind separate clouds with nothing in between. Then, of course, the expansion is still more rapid: one does not need diffusion, since there are expansion waves. Oort calculated a time scale from the expansion in connection with the distances between the clouds, and thus found times of the order of a few millions of years. This was the same scale as had been obtained from other considerations. Diffusion and thermal conduction can be important accompanying factors, but I would not believe that they will change the picture materially.

F. D. KAHN : Not everyone would agree that all HI clouds are surrounded by an HII region. This latter, I suppose, was introduced in order to keep the pressures equal inside and outside the clouds, but since we are talking about a medium in a state of supersonic turbulence, the equality is not really necessary. It is quite possible that the HI clouds are surrounded by a very tenuous, cool neutral gas.

H. C. VAN DE HULST: Whether there is actual pressure equality in space anywhere at the boundary between HI and HII, I do not want to pronounce a judgment, although I would rather think there is not. But if we ask whether the following situation could persist; an HI region with a low temperature and high density and an HII region with high temperature and low density, with the same pressure, and with ionizing radiation continuously falling on the interface from the 
HII side and cooling mechanisms sufficient to do the normal cooling, then the answer is that it can persist indefinitely. ${ }^{5}$ The question of diffusion just does not arise because any HI coming across the interface is rapidly ionized and any HII going into the cool region rapidly recombines, which does not enter into the energy equation.

M. MINNAERT, Sterrewacht Sonnenburg, Utrecht, Netherlands: On the last day of this symposium it may be allowed to try to construct an oversimplified model of galactic space. I am thinking especially about the space around the plane of the galactic equator. We know that, around the very hot stars, we have to assume HII regions, which according to an old estimate fill about $1 / 10$ of space. In between what will there be? We cannot say that all remaining parts of space are normal HI regions - at least if we are of the opinion that the HI clouds are distinct clouds separated by big spaces of very low density. I know there is also another current of thought, but I had the impression that many of us have been convinced by the photographs of the multiple components of interstellar lines. There must be something in between which is cold, and has an extremely low density, "almost nothing." Let us call that HIII regions. Now is it possible to give an estimate of the ratios between the three regions, HI, HII, and HIII? Or is there another oversimplified picture which is better?

L. SPITZER, JR. : Let me describe the picture that I would personally draw if asked to construct a simplified cloud model, realizing, of course, that no cloud model can really explain the observations. First, I would draw the scattered dense clouds, mostly cool and neutral, roughly $10 \%$ by volume of space. Second, I would draw in a number of $\mathrm{O}$ stars around the neighborhood. These stars are of various ages and are embedded in interstellar material of quite different densities, so the HII regions around them have different appearances. Around some of the stars we observe nearly circular loops of dense HII: such a system is presumably a dense cloud, formerly cool, but now hot, and expanding rapidly. Possibly ten percent of the volume in the spiral arms may be occupied by these dense HII regions. Around some of the $\mathrm{O}$ stars, we may expect rarified HII regions, so rarified that one cannot detect any emission lines at all. This agrees with the observation that some $O$ stars seem to have no material around them. In between these HII regions, surrounding individual $\mathrm{O}$ stars, we shall find quite generally the ultraviolet light emanating from those $\mathrm{O}$ stars which are not embedded in dense regions. This light, which may be multiply scattered, penetrates the whole intercloud region which Minnaert has termed HIII, but which might well be actually HII or even $\mathrm{HI}$ in some spots.

${ }^{5}$ This is the $D$-critical case discussed by F. D. Kahn [Bull. Astron. Soc. Neth. 12, No. 456, 187 (1954)] and Pottasch [Bull. Astron. Soc. Neth. (to be published)].
In any case, this intercloud medium is probably at a high temperature, so that its pressure is equal to that in the denser, cooler HI regions. Whether this rarified gas between clouds, and not near an early-type star, is HII or HI is an open question.

A. SCHLÜTER, Max Planck Institut für Physik, Göttingen, Germany: I would only give $1 \%$ of space to the dense HII clouds. With respect to the real ionization of the hot gas, it seems reasonable to assume it ionized ; for only a little ultraviolet radiation is needed to keep it so, since the density is low and therefore the recombination time is very long.

G. C. MCVITTIE, University of Illinois, Urbana, Illinois: Is my impression correct: around some $\mathrm{O}$ stars you have not observed any material, but you feel there ought to be some present?

L. SPITZER, JR. : It is correct to say that there is no gas actually observed around some of these O-type stars, and having said a little earlier that we don't understand the dynamics of interstellar material, I am not in a good position now to make a firm theoretical statement about the density of gas there. However, I would assume that the pressure in this region is not many orders of magnitude less than the pressure in the surrounding region.

G. B. FIELD : It seems to me that it would be relevant to this discussion to note the marvellous map of the $\mathrm{HI}$ emission in the galaxy, recently compiled by the Netherlands astronomers in Bull. Astron. Soc. Neth., No. 475. You see the spiral arm structure, but also you see that in a few places the derived density of $\mathrm{HI}$ drops to zero. Between the arms we find between $1 / 20$ and $1 / 4$ of an atom per $\mathrm{cm}^{3}$. So I would argue that in fact what has been called HIII contains a significant proportion of HI.

L. SPITZER, JR.: I would assume that this map represents the mean density over a region of considerable extent. Hence, I believe that these low density regions may contain a number of $\mathrm{HI}$ clouds. Incidentally, I hope that the term HIII does not become a standard notation for the intercloud medium. Spectroscopically such a designation might be a little confusing.

M. MinnaERT: This gives me the opportunity to remark how horrible to spectroscopists is the notation of "HI and HII regions." The symbol HI, means the spectrum of the hydrogen atom; and HII ought to designate the spectrum of the hydrogen ion, which however does not exist. I think that any spectroscopist would prefer to speak about $\mathrm{H}$ regions and $\mathrm{H}^{+}$regions.

With these remarks the discussion came to an end. After this, Professor Redman from Cambridge, England, thanked the hosts in the name of the foreign participants of the Symposium; a telegram of greetings was sent to Professor J. H. Oort in Leiden; and Professor Burgers closed the meeting with thanks to the recording staff. 